\title{
A new quasi-newton equation on the gradient methods for optimization minimization problem
}

\author{
Basim A. Hassan, Ghada M. Al-Naemi \\ Department of Mathematics, College of Computers Sciences and Mathematics, University of Mosul, Iraq
}

\begin{tabular}{l} 
Article Info \\
\hline Article history: \\
Received Jan 1, 2020 \\
Revised Mar 4, 2020 \\
Accepted Mar 18, 2020 \\
\hline
\end{tabular}

\section{Keywords:}

Global convergence

Quasi-newton equation

Quasi-newton method

\begin{abstract}
The quasi-Newton equation is the very foundation of an assortment of the quasi-Newton methods for optimization minimization problem. In this paper, we deriving a new quasi-Newton equation based on the second-order Taylor's series expansion. The global convergence is established underneath suitable conditions and numerical results are reported to show that the given algorithm is more effective than those of the normal BFGS method.
\end{abstract}

\section{Corresponding Author:}

Basim A. Hassan,

Department of Mathematics,

College of Computers Sciences and Mathematics,

University of Mosul, Iraq.

Email: basimah@uomosul.edu.iq, basimabas39@gmail.com

\section{INTRODUCTION}

The quasi-Newton algorithm is one of the more successful algorithms for unconstrained nonlinear programming [1]. These methods, which use the updating formulas for approximation of the Hessian. To minimize a multi-variable nonlinear function this our objective :

$$
\operatorname{Min} f(x), \mathrm{x} \in \mathrm{R}^{\mathrm{n}}
$$

where $f$ is twice differentiable. Throughout the paper, we define by $B_{k}$ is a nonnegative definite matrix that estimates the Hessian matrix $Q_{k}=\nabla^{2} f\left(x_{k}\right)$ of $f\left(x_{k}\right)$ and $g_{k}=\nabla f\left(x_{k}\right)$ is the gradient of $f\left(x_{k}\right)$. More details can be found in [2]. For most optimization algorithms, the search for the minimizer of (1) is carried out by using :

$$
x_{k+1}=x_{k}+\alpha_{k} d_{k}
$$

where $\alpha_{k}$ is the step size, and $d_{k}$ is the search direction. Moreover, the search direction $d_{k}$ of the quasi-Newton methods often has the form :

$$
B_{k} d_{k}+g_{k}=0
$$


It estimates $B_{k}$ update formula, we will focus on the BFGS method which has proved to be the most effective of all quasi-Newton methods :

$$
B_{k+1}^{B F G S}=B_{k}-\frac{B_{k} s_{k} s_{k}^{T} B_{k}{ }^{T}}{s_{k}^{T} B_{k} s_{k}}+\frac{y_{k} y_{k}^{T}}{s_{k}^{T} y_{k}}
$$

It's also well known that the matrix $B_{k+1}$ is generated by (4) to satisfy the secant equation :

$$
B_{k+1} s_{k}=y_{k}
$$

where $s_{k}=x_{k+1}-x_{k}=\alpha_{k} d_{k}$ and $y_{k}=g_{k+1}-g_{k}$, for more details see [3, 4].

It is easy to take advantage of the following relationships : $B_{k+1} s_{k} \Leftrightarrow y_{k}, B_{k} \Leftrightarrow H_{k}$ where $B_{k}^{-1}=H_{k}$, then the famous BFGS formula is designed by :

$$
H_{k+1}^{B F G S}=H_{k}-\frac{H_{k} y_{k} s_{k}^{T}+s_{k} y_{k}^{T} H_{k}}{s_{k}^{T} y_{k}}+\left[1+\frac{y_{k}^{T} H_{k} y_{k}}{s_{k}^{T} y_{k}}\right] \frac{s_{k} s_{k}^{T}}{s_{k}^{T} y_{k}}
$$

For details see [5]. When the general function no one has proved the convergence property of the BFGS method. To acquire improved quasi-Newton methods, many modified quasi-Newton equations have been proposed ([6-10], among others) and established its convergence property. Using second-order Taylor's series approximation of the function to derivation the a new quasi-Newton equation and we study convergence property and numerical results.

\section{DERIVING QUASI-NEWTON EQUATIONS} is defined by :

We deriving new quasi-Newton equations based on the second-order Taylor's series expansion and

$$
f\left(x_{k}\right)=f\left(x_{k+1}\right)-g_{k+1}^{T} s_{k}+\frac{1}{2} s_{k}^{T} Q s_{k}
$$

Using exact line search, we get :

$$
f\left(x_{k}\right)=f\left(x_{k+1}\right)+\frac{1}{2} s_{k}^{T} Q s_{k}
$$

which implies that :

$$
s_{k}^{T} Q s_{k}=2\left(f\left(x_{k}\right)-f\left(x_{k+1}\right)\right)
$$

Add and subtract from the right tip the $s_{k}^{T} y_{k}$, we get :

$$
s_{k}^{T} Q s_{k}=2\left(f\left(x_{k}\right)-f\left(x_{k+1}\right)\right)+s_{k}^{T} y_{k}-s_{k}^{T} y_{k}
$$

The choice $B_{k+1}$ is key to the approximate effect of the Hessian matrix $Q$ :

$$
s_{k}^{T} B_{k+1} s_{k}=2\left(f\left(x_{k}\right)-f\left(x_{k+1}\right)\right)+s_{k}^{T} y_{k}-s_{k}^{T} y_{k}
$$

which implies a new QN equation and as follows : 


$$
B_{k+1} s_{k}=\tilde{y_{k}}, \quad \tilde{y}_{k}=y_{k}+\frac{2\left(f\left(x_{k}\right)-f\left(x_{k+1}\right)\right)-s_{k}^{T} y_{k}}{s_{k}^{T} v_{k}} v_{k}
$$

where $v_{k}$ is any vector such that $s_{k}^{T} v_{k} \neq 0$. Now we Applying the a new quasi-Newton equation in the two cases :

Case i : If $v_{k}=y_{k}$, we get :

$$
\tilde{y_{k}}=y_{k}+\frac{2\left(f\left(x_{k}\right)-f\left(x_{k+1}\right)\right)-s_{k}^{T} y_{k}}{s_{k}^{T} y_{k}} y_{k}
$$

Case ii : If $v_{k}=g_{k+1}$, we get :

$$
\tilde{y_{k}}=y_{k}+\frac{2\left(f\left(x_{k}\right)-f\left(x_{k+1}\right)\right)-s_{k}^{T} y_{k}}{s_{k}^{T} g_{k+1}} g_{k+1}
$$

The new algorithm can be staged formally as follows.

\section{New Algorithm :}

Stage 1 : select $x_{0} \in R^{n}$ and $H_{0}=I$. Set $k=0$.

Stage 2 : Stop if $\left\|g_{k}\right\|=0 . s_{k}^{T} \tilde{y_{k}}>0$

Stage 3 : Generate $d_{k}$ by $d_{k}=-H_{k} g_{k}$.

Stage 4 : Find a $\alpha_{k}$ based on the Wolfe condition satisfies :

$$
\begin{aligned}
& f\left(x_{k}+\alpha_{k} d_{k}\right) \leq f\left(x_{k}\right)+\delta \alpha_{k} g_{k}^{T} d_{k} \\
& d_{k}^{T} g\left(x_{k}+\alpha_{k} d_{k}\right) \geq \sigma d_{k}^{T} g_{k}
\end{aligned}
$$

Stage 5 : Variable update, $x_{k+1}=x_{k}+\alpha_{k} d_{k}$.

Stage 6 : If $s_{k}^{T} \tilde{y}{ }_{k}>0$, find update $H_{k+1}$ by the formula (6) and (12), otherwise let $H_{k+1}=H_{k}$. stage 7 : Let $k=k+1$. Go to stage 2 .

We verify the positive definite property of the update formula for the quasi-Newton method in the next theorem.

\section{Theorem 1.}

If and if only $s_{k}^{T} \tilde{y_{k}}>0$, then the update $H_{k+1}$ is positive definite.

\section{Proof :}

The gradient-difference vector $y_{k}$, we define by :

$$
\tilde{y_{k}}=y_{k}+\frac{2\left(f\left(x_{k}\right)-f\left(x_{k+1}\right)\right)-s_{k}^{T} y_{k}}{s_{k}^{T} v_{k}} v_{k}
$$

Multiplying above equation by $s_{k}^{T}$, we obtain : 
$s_{k}^{T} \tilde{y_{k}}=s_{k}^{T} y_{k}+2\left(f\left(x_{k}\right)-f\left(x_{k+1}\right)\right)-s_{k}^{T} y_{k}$

From above equation we get :

$s_{k}^{T} \tilde{y_{k}}=2\left(f\left(x_{k}\right)-f\left(x_{k+1}\right)\right)$

Using first Wolfe condition in above equation, we obtain :

$s_{k}^{T} \tilde{y_{k}} \geq-2 \delta \alpha_{k} g_{k}^{T} d_{k}$

In fact, $s_{k}^{T} g_{k}=\alpha_{k} d_{k}^{T} g_{k}<0$, such that :

$s_{k}^{T} \tilde{y_{k}} \geq-2 \delta \alpha_{k} d_{k}^{T} g_{k}>0$

The proof is complete.

\section{CONVERGENCE ANALYSIS} methods.

Now by using under the following assumption, we twist to the convergence result of the new

\section{Assumptions :}

a) $f$ is bounded on the set $S=\left\{x \in R^{n} \mid f(x) \leq f\left(x_{0}\right)\right\}$ and is bounded below on $R^{n}$.

b) If there exits a nonnegative constant $L$ such that :

$\|g(z)-g(w)\| \leq L\|z-w\|, \quad \forall z, w \in R$

and $g$ is called Lipschitz continuous and we get :

$\|g(x)\| \leq \gamma, \quad \forall x \in R$

If $\left\{f\left(x_{k}\right)\right\}$ is a decreasing, then $\left\{x_{k}\right\}$ is contained in $R$ and the existence of $x^{*}$ we get:

$\lim _{k \rightarrow \infty} f\left(x_{k}\right)=f\left(x^{*}\right)$

In veracity, that sequence $x_{k}$ is restricted, there exist some positive constant $\mu$ such that :

$$
\left\|s_{k}\right\|=\|x-\bar{x}\| \leq\|x\|+\|-\| x \| \leq \mu
$$

For more details see [11].

\section{Theorem 2.}

If the following inequality holds :

$$
\left\|B_{k} s_{k}\right\| \leq a_{1}\left\|s_{k}\right\| \quad \text { and } s_{k}^{T} B_{k} s_{2} \geq a_{2}\left\|s_{k}\right\|^{2}
$$

where $a_{1}>0$ and $a_{2}>0$ are constants and the $\left\{x_{k}\right\}$ be generated by new methods. For infinitely $k$, then we get: 
$\liminf _{k \rightarrow \infty}\left\|g_{k}\right\|=0$

Proof :

Using (26) and adding with $g_{k}=-B_{k} d_{k}$, we gives :

$a_{2}\left\|d_{k}\right\| \leq\left\|g_{k}\right\| \leq a_{1}\left\|d_{k}\right\| \quad$ and $\quad d_{k}^{T} B_{k} d_{2} \geq a_{2}\left\|d_{k}\right\|^{2}$

The two conditions form Wolfe rule (15), (16) and (28) to obtain :

$-(1-\sigma) g_{k}^{T} d_{k} \leq\left(g_{k+1}-g_{k}\right)^{T} d_{k} \leq L \alpha_{k}\left\|d_{k}\right\|^{2}$

Then (29) implies that :

$\alpha_{k} \geq \frac{-(1-\sigma) g_{k}^{T} d_{k}}{L\left\|d_{k}\right\|^{2}}=\frac{(1-\sigma) d_{k}^{T} B_{k} d_{k}}{L\left\|d_{k}\right\|^{2}} \geq \frac{(1-\sigma) a_{2}}{L}$

Accordingly (24), we have :

$\sum_{k=1}^{\infty}\left(f_{k}-f_{k+1}\right)=\lim _{N \rightarrow \infty} \sum_{k=1}^{\infty}\left(f_{k}-f_{k+1}\right)=\lim _{N \rightarrow \infty}\left(f_{1}-f_{k+1}\right)=f_{1}-f^{*}$

from which it follows that :

$\sum_{k=1}^{\infty}\left(f_{k}-f_{k+1}\right) \leq+\infty$

merge them with Wolfe law (15) leads :

$\sum_{k=1}^{\infty}-\alpha_{k} g_{k}^{T} d_{k} \leq+\infty$

We obtained :

$\lim _{k \rightarrow \infty} \alpha_{k} g_{k}^{T} d_{k}=0$

above equally with (34) give that :

$\lim _{k \rightarrow \infty} d_{k}^{T} B_{k} d_{k}=\lim _{k \rightarrow \infty}-g_{k}^{T} d_{k}=0$

Merge (35) with (28) we get the finale (27). If $f$ is non-convex function, we need some assumptions on the update, may can lose the positive definiteness. For every $k$, we define the index set $K$

$$
K=\left\{k: \frac{s_{k}^{T} \overline{y_{k}}}{\left\|s_{k}\right\|} \geq \beta\left\|g_{k}\right\|^{\delta}\right\},
$$

where $\beta>0$ is constant and $\delta>0$ is bounded. The next lemma quoted in [12] is very significant to study the convergence property. 


\section{Lemma 1.}

If BFGS method with Wolfe condition is applied to a continuously differentiable function $f$ that is bounded below, and if there exists a constant $M$ such that the inequality holds :

$$
\frac{\left\|y_{k}\right\|^{2}}{s_{k}^{T} y_{k}} \leq M
$$

then :

$$
\lim _{k \rightarrow \infty} \inf \left\|g_{k}\right\|=0
$$

A cautious update rule similar to the above lemma.

\section{Theorem 3.}

Suppose $\left\{x_{k}\right\}$ be generated by the new method. Then we get :

$$
\lim _{k \rightarrow \infty} \inf \left\|g_{k}\right\|=0
$$

\section{Proof :}

In view of Theorem 2., sufficiently show that (26) holds for infinitely k. If $K$ is a finite set, then $B_{k}$ is a constant-matrix, obviously, (26) satisfies. Now, if $K$ is a infinite we will deduce a contradiction with there exists $\varepsilon>0$ such that :

$$
\left\|g_{k}\right\|>\varepsilon
$$

It follows from (36), we obtain :

$$
s_{k}^{T} \tilde{y}_{k} \geq \beta \varepsilon^{\delta}\left\|s_{k}\right\|
$$

Using definition of $\tilde{y}_{k}$, we have :

$$
\begin{aligned}
\left\|\tilde{y}_{k}\right\| & =\left\|y_{k}+\frac{2\left(f_{k}-f_{k+1}\right)-s_{k}^{T} y_{k}}{s_{k}^{T} v_{k}} v_{k}\right\| \\
& \leq\left\|y_{k}\right\|
\end{aligned}
$$

It follows from (22) and (42) we obtain :

$$
\left\|\tilde{y}_{k}\right\| \leq\left\|y_{k}\right\| \leq L\left\|s_{k}\right\|
$$

This, together with (41), lead to :

$$
\frac{\left\|\tilde{y_{k}}\right\|^{2}}{s_{k}^{T} \tilde{y}_{k}} \leq M
$$


Applying lemma 1, to the $\left\{B_{k}\right\}_{k \in K}$, there exist, $a_{1}$ and $a_{2}$, we obtain (21) for infinitely many $k$. Then, proof is finished.

\section{NUMERICAL EXPERIMENTS}

It has been programmed subroutine Matlab to test the modified BFGS algorithm presented in the previous section. We tested the algorithm on the following problems that have been taken from [13]. Different test functions have been used in different researchs such as [14-24].

All the problems are being resolved successfully, and numbers are given duplicates and job evaluation in Table 1. We have solved these problems through the BFGS algorithm, as shown in Table 1 the numerical results of the new algorithm. The Himmeblau [25], stop rule is used : If $\left|f\left(x_{k}\right)\right|>10^{-5}$, let stop $1=\left|f\left(x_{k}\right)-f\left(x_{k+1}\right)\right| /\left|f\left(x_{k}\right)\right| ; \quad$ Otherwise, let stop $1=\left|f\left(x_{k}\right)-f\left(x_{k+1}\right)\right|$. For every problem, if $\left\|g_{k}\right\|<\varepsilon$ or stop $1<10^{-5}$ is satisfied, the program will be stopped. To compare the efficiency of roads in Table 1, we adopt the number of iterations (NI) and the number of evaluations of jobs (NF). Numerically results show that the new algorithm is a little better than the usual BFGS algorithm in this group of test problems.

Table 1. Numerical results

\begin{tabular}{|c|c|c|c|c|c|c|c|}
\hline \multirow[b]{2}{*}{ Problems } & \multirow[b]{2}{*}{$\mathrm{n}$} & \multicolumn{2}{|c|}{ BFGS algorithm } & \multicolumn{2}{|c|}{ BFGS with $v_{k}=y_{k}$} & \multicolumn{2}{|c|}{ BFGS with $v_{k}=g_{k+1}$} \\
\hline & & NI & $\mathrm{NF}$ & NI & NF & NI & $\mathrm{NF}$ \\
\hline Froth & 2 & 9 & 26 & 9 & 26 & 11 & 32 \\
\hline Badscp & 2 & 43 & 166 & 34 & 125 & 3 & 31 \\
\hline Badscb & 2 & 3 & 30 & 3 & 30 & 3 & 30 \\
\hline Beale & 2 & 15 & 50 & 13 & 43 & 13 & 38 \\
\hline Jensam & 2 & 2 & 27 & 2 & 27 & 2 & 27 \\
\hline Helix & 3 & 34 & 113 & 24 & 80 & 17 & 49 \\
\hline Bard & 3 & 16 & 54 & 17 & 56 & 12 & 36 \\
\hline Gauss & 3 & 2 & 4 & 2 & 4 & 2 & 4 \\
\hline Gulf & 3 & 2 & 27 & 2 & 27 & 2 & 27 \\
\hline Box & 3 & 2 & 27 & 2 & 27 & 2 & 27 \\
\hline Sing & 4 & 20 & 60 & 14 & 44 & 11 & 35 \\
\hline Wood & 4 & 19 & 61 & 19 & 61 & 7 & 22 \\
\hline Kowosb & 4 & 21 & 65 & 23 & 117 & 10 & 28 \\
\hline $\mathrm{Bd}$ & 4 & 17 & 54 & 16 & 50 & 8 & 27 \\
\hline Osb1 & 5 & 2 & 27 & 2 & 27 & 2 & 27 \\
\hline Biggs & 6 & 25 & 72 & 8 & 50 & 9 & 27 \\
\hline Osb2 & 11 & 3 & 31 & 3 & 31 & 3 & 31 \\
\hline Watson & 20 & 31 & 102 & 34 & 108 & 6 & 20 \\
\hline Rosex & 100 & 231 & 806 & 46 & 197 & 33 & 765 \\
\hline Singx & 400 & 64 & 209 & 126 & 397 & 10 & 32 \\
\hline Pen1 & 400 & 2 & 27 & 2 & 27 & 2 & 27 \\
\hline Pen2 & 200 & 2 & 5 & 2 & 5 & 2 & 5 \\
\hline Vardi & 100 & 2 & 27 & 2 & 27 & 2 & 27 \\
\hline Trig & 500 & 9 & 33 & 8 & 28 & 14 & 42 \\
\hline $\mathrm{Bv}$ & 500 & 2 & 4 & 2 & 4 & 2 & 4 \\
\hline Ie & 500 & 6 & 16 & 7 & 19 & 13 & 37 \\
\hline Band & 500 & 57 & 281 & 15 & 81 & 5 & 16 \\
\hline Lin & 500 & 2 & 4 & 2 & 4 & 2 & 4 \\
\hline Lin1 & 500 & 3 & 7 & 3 & 7 & 3 & 7 \\
\hline Lin0 & 500 & 3 & 7 & 3 & 7 & 3 & 7 \\
\hline Total & & 649 & 2422 & 445 & 1736 & 214 & 1491 \\
\hline
\end{tabular}

Computational results show that a reduction of (31-67)\% and (28-38)\% in terms of the total number of iterations and function evaluations respectively. Relative efficiency of the new algorithms as shown in Table 2.

Table 2. Relative efficiency of the new algorithms

\begin{tabular}{cccc}
\hline & BFGS algorithm & BFGS with $v_{k}=y_{k}$ & BFGS with $v_{k}=g_{k+1}$ \\
\hline $\mathrm{NI}$ & $100 \%$ & $68.56 \%$ & $32.97 \%$ \\
$\mathrm{NF}$ & $100 \%$ & $71.67 \%$ & $61.56 \%$ \\
\hline
\end{tabular}




\section{CONCLUSIONS}

In this paper, we deriving a new quasi-Newton equation based on the second-order Taylor's series approximation of the function. We conclude by affirming that the arithmetical findings in this work was effective to solving optimization problems.

\section{ACKNOWLEDGMENT}

The authors are very grateful to the University of Mosul / College of Computers Sciences and Mathematics for their provided facilities, which helped to improve the quality of this work.

\section{REFERENCES}

[1] D. P. Bertsekas, "Nonlinear Programming," Belmont, Massachusetts, Athena Scientific, 1996.

[2] G. Yuan and Z. Wei, "Convergence analysis of a modified BFGS method on convex minimizations," Computational Optimization and Applications, vol. 47, pp. 237-255, 2010.

[3] Y. Dai, "Convergence properties of the BFGS algorithm," SIAM Journal on Optimization, vol. 13, no. 3, pp. 693-701, 2002.

[4] Y. Xiao and Z. Wei, "A Modified BFGS Method Without Line Searches for Nonconvex Unconstrained Optimization," Advances in Theoretical and Applied Mathematics, vol. 1, pp. 149-162, 2006.

[5] G. Yuan, et al., "The global convergence of a modified BFGS method for nonconvex functions," Journal of Computational and Applied Mathematics, vol. 327, pp. 274-294, 2018.

[6] F. Biglari, et al., "New quasi-Newton methods via higher order tensor models," Journal of Computational and Applied Mathematics, vol. 235, no. 8, pp. 2412-2422, 2011.

[7] L. H. Chen, et al., "A modified quasi-Newton method for structured optimization with partial information on the Hessian," Computational Optimization and Applications, vol. 35, pp. 5-18, 2006.

[8] Z. Wei, et al., "New quasi-Newton methods for unconstrained optimization problems," Applied Mathematics and Computation, vol. 175, no. 2, pp. 1156-1188, 2006.

[9] Basim A. H., "A modified quasi-Newton methods for unconstrained Optimization," Journal of pure and applied mathematics, vol. 42, pp. 504-511, 2019.

[10] Basim A. H. and Hussein K. K., "A new class of BFGS updating formula based on the new quasi-newton equation," Indonesian Journal of Electrical Engineering and Computer Science, vol. 13, no. 3, pp. 945-953, 2019.

[11] R. Dehghani, et al., "The modified quasi-Newton methods for solving unconstrained optimization problems," International Journal of Numerical Modelling: Electronics Networks, Devices and Fields, vol. 32, no. 1, pp. 1-8, 2018.

[12] M. J. D. Powell, "Some global convergence properties of a variable metric algorithm for minimization without exact line searches," in R. W. Cottle and C. E. Lemke, eds, Nonlinear Programming, SIAM-AMS Proceedings, vol. 4, pp. 53-72, 1976.

[13] J. J. More, et al., "Testing unconstrained optimization software," ACM Transactions on Mathematical. Software, vol. 7, no. 1, pp. 17-41, 1981 .

[14] B. A. Hassan and H. N. Jabbar, "A New Transformed Biggs 's Self-Scaling Quasi-Newton Method for Optimization," Zanco Journal of Pure and Applied Sciences, vol. 31, no. 2, pp. 1-5, 2019.

[15] R. H. Byrd and J. Nocedal, "A tool for the analysis of quasi-Newton methods with application to unconstrained minimization," SIAM Journal on Numerical Analysis, vol. 26, no. 3, pp. 727-739, 1989.

[16] Basim A. H., et al., "A descent extension of the Dai - Yuan conjugate gradient technique," Indonesian Journal of Electrical Engineering and Computer Science, vol. 16, no. 2, pp. 661-668, 2019.

[17] Basim A. H., "A new type of quasi-Newton updating formulas based on the new quasi-Newton equation," Numerical Algebra, Control and Optimization, vol. 10, no. 2, pp. 227-235, 2020.

[18] Basim A. H., et al., "A new kind of parameter conjugate gradient for unconstrained optimization," Indonesian Journal of Electrical Engineering and Computer Science, vol. 17, no. 1, pp. 404-411, 2020.

[19] Basim A. H., et al., "A Class of Descent Conjugate Gradient Methods for Solving Optimization Problems," Applied Mathematical Sciences, vol. 13, no. 12, pp. 559-567, 2019.

[20] Basim A. H. and Mohammed W. T., "A Modified Quasi-Newton Equation in the Quasi-Newton Methods for Optimization," Applied Mathematical Sciences, vol. 13, no. 10, pp. 463-472, 2019.

[21] Basim A. H., "A Globally Convergence Spectral Conjugate Gradient Method for Solving Unconstrained Optimization Problems," Al-Rafidain Journal of Computer Sciences and Mathematics, vol. 10, no. 4, pp. 21-28, 2013.

[22] Basim A. H., "Development a Special Conjugate Gradient Algorithm for Solving Unconstrained Minimization Problems," Al-Rafidain Journal of Computer Sciences and Mathematics, vol. 9, no. 1, pp. 73-84, 2012.

[23] Basim A. H. and Omer M. E., "A New sufficient descent Conjugate Gradient Method for Nonlinear Optimization," Iraqi Journal of Statistical Sciences, vol. 14, no. 26, pp. 12-24, 2014.

[24] Basim A. H., "A new formula for conjugate parameter computation based on the quadratic model," Indonesian Journal of Electrical Engineering and Computer Science, vol. 13, no. 3, pp. 954-961, 2019.

[25] Y. Yuan and W. Sun, "Theory and Methods of Optimization,” Science Press of China, 1999. 